(C) 2015 Optical Society of America. One print or electronic copy may be made for personal use only. Systematic reproduction and distribution, duplication of any material in this paper for a fee or for commercial purposes, or modifications of the content of this paper are prohibited.

Available online at:

https://www.osapublishing.org/ao/abstract.cfm?uri=ao-54-36-10717 


\title{
Carrier-envelope phase changes in the focal region: propagation effects measured by spectral interferometry
}

\author{
Balázs Major ${ }^{1,}{ }^{*}$, Dániel Nemes ${ }^{1}$, Miguel A. Porras ${ }^{2}$, Zoltán L. Horváth ${ }^{1}$, and Attila P. \\ KOVÁCS ${ }^{1}$ \\ ${ }^{1}$ Department of Optics and Quantum Electronics, University of Szeged, Dóm tér 9., Szeged H-6720, Hungary \\ ${ }^{2}$ Grupo de Sistemas Complejos, Universidad Politécnica de Madrid, Rios Rosas 21, Madrid ES-28003, Spain \\ "Corresponding author: bmajor@titan.physx.u-szeged.hu
}

Compiled January 5, 2016

\begin{abstract}
Spectral interferometric measurements are presented which show how wave propagation affects the carrier-envelope phase (CEP) of an ultrashort pulse in the focal region, and results in variations that are different from the Gouy phase shift. Wavelength-dependent properties of the input beam are investigated and are seen to influence how the CEP is altered. The measured CEP changes show characteristics similar to the variations predicted by theory. @ 2016 Optical Society of America
\end{abstract}

OCIS codes: (120.3180) Interferometry; (140.3295) Laser beam characterization; (260.7120) Ultrafast phenomena; (320.7100) Ultrafast measurements

http://dx.doi.org/10.1364/AO.54.010717

\section{INTRODUCTION}

As a result of the continuous technological development of laser systems, electromagnetic wave packets formed by only a few cycles of the carrier wave are routinely obtainable for a wide range of scientific studies [1]. In the last decade even single- or subcycle optical wave forms became available in laser laboratories [2]. If pulses are such short in duration not only the temporal envelope is relevant, the outcome of the experiment is instead determined by how the electric field itself changes in time [1]. This recognition initiated the demand for the phase-stable production of ultrashort pulses [3], i.e., of producing pulses with stable phase shot-to-shot using the available laser oscillators, amplifiers or wave-form synthesizers [2].

Even with the current highly energetic, precisely shaped and reproducible electromagnetic pulses one thing is almost always necessary for the desired phenomena to occur: focusing of the beam. Due to focusing, however, the carrier-envelope phase (CEP) is known to change during propagation through the focal region [4-9], and this change can differ from Gouy phase shift [5, 7-9]. This can be relevant in CEP-dependent effects where the interaction of light and matter takes place in a larger volume, as in high harmonic generation [10], above threshold ionization of gases [11], photodissociation of molecules [12], multiphoton ionization [13] or in methods for absolute phase determination [14].

In this work we use Fourier-transform spectral interferometry
(FTSI) [15] to study how the CEP changes when a focused, short optical pulse propagates through focus. While there are previous experimental studies on these phase variations [4-6], they only report changes similar to the Gouy phase shift [16]. However, it has been shown theoretically that the different propagation behavior of the different monochromatic components of the pulsed beam can relevantly alter the CEP in the focal region $[9,17]$. These CEP variations - different from Gouy phase shift - are measured for the first time - up to our knowledge - in this work. An experimental study of the wavelength-dependent beam-propagation properties affecting the CEP is presented here, leading to the particular CEP changes. The results highlight the general importance of these phase-affecting pulsed-beam features, which are often neglected and the effect of which can appear not just when lenses are used, but also when a mirror is the focusing element [17]. The measured data exhibit variations similar to those obtained from numerical simulations.

The present work is structured as follows. First, the theoretical background of the analyzed CEP phenomenon is discussed highlighting the most important features related to the current study. Then the theory behind the FTSI measurement method is detailed. After introducing the experimental setup used, the last part of the Experimental section deals with the evaluation of the interferometric data, listing also the possible sources of error for the measurements carried out. In the Results section, after describing the beam characterization process, both the beam 
characteristics and the CEP changes are shown for the particular case presented in this work. Finally, the most important conclusions are drawn.

\section{THEORETICAL BACKGROUND}

\section{A. On-axis changes of the carrier-envelope phase}

The Gouy phase shift of monochromatic beams in the focus is a well-known property [16]. For pulsed beams, which consist of a broad spectrum of harmonic waves, a similar change for the CEP is expected [1,4]. However, as the variation of the CEP during propagation is a result of the difference between the phase- and the group velocity, a CEP change like Gouy phase shift is only typical for pulsed beams with certain properties. Limiting the considerations to cases when the phase-velocity variation is purely determined by the Gouy phase shift, the CEP can follow the same curve only if the group velocity is constant during the travel through focus. In the case of Gaussian beams this is the unique feature of a focused beam that is isodiffracting (i.e., all monochromatic components have the same Rayleigh range) and all harmonic components have their waists at the same plane perpendicular to the optical axis [17]. However, this is not the general case.

Variations in the group velocity, and as a result changes in the $\mathrm{CEP}$, are originated from two wavelength dependent properties of Gaussian beams. On the one hand, if the beam waist size of the broadband beam changes such a way that the Rayleigh length is not the same for each component, the phase delay obtained during propagating a given distance will be different for each color, resulting in a group velocity modification. Similarly, if the waists of the various components are at distinct positions, a group velocity change occurs [17].

If one considers a pulsed Gaussian beam which is focused at its waist, it has been shown that the on-axis CEP change relative to its value measured in the focal point follows the formula [9]

$$
\Delta \Phi(\xi)=-\operatorname{atan}(\xi)+g \frac{\xi}{1+\xi^{2}}+\gamma \frac{\xi^{2}}{1+\xi^{2}} .
$$

In the expression above $\xi=z / L_{0}$ is the distance $z$ measured from the geometrical focus of the carrier wave in the units of the focused Rayleigh length $L_{0}$. Subscript zero means evaluation at the carrier angular frequency $\omega_{0}$. The parameters $g$ and $\gamma$ characterize the frequency dependence of the beam size and the same feature for the focal length of the lens, respectively. Expression (1) shows intuitively how the mentioned properties of Gaussian beams, and as such the propagation properties, induce CEP changes different from Gouy phase shift. It is important that formula (1) assumes that the pulse shape does not change during the propagation through focus. It is also presumed that if a lens is used as a focusing element, its material dispersion is pre-compensated (no relevant second- or higher order phase derivative is present). The CEP variations predicted by (1) with the above requirements are independent of pulse duration, valid either for few-, many-cycle or transform-limited pulses [9].

While in the original interpretation of (1) the focusing element was placed strictly at the waist of the source beam, the expression can be generalized. Originally the value of parameter $g$ originated from the wavelength dependence of source beam waist $s$, according to

$$
g=1+\left.2 \frac{\partial s}{\partial \omega}\right|_{\omega_{0}} \frac{\omega_{0}}{s_{0}} .
$$

However, as (1) can be deduced from diffraction theory [9], $s$ does not need to be the waist size of the source beam. Generally, the beam size at the focusing element can be used instead as $s$ in (2) when the position of the waist does not coincide with the place of the focal element [17].

When the beam is focused at its waist, parameter $\gamma$ in (1) is purely defined by the chromatic aberration of the optical element used for focusing. This means that the paraxial focal length $f$ changes with wavelength, and the parameter is given by

$$
\gamma=\left.\frac{\partial f}{\partial \omega}\right|_{\omega_{0}} \frac{\omega_{0}}{L_{0}} .
$$

Considering again the general formulas of diffraction, on which (1) is based, $f$ is the paraxial wave-front curvature behind the focusing element in (3) [18]. It is known that the focused wavefront curvature is not defined solely by the focal length, but by the wave-front radius before the focusing element and the focal length together [17]. With the knowledge of these two quantities, the paraxial wave-front curvature $f$ and its frequency dependence can be obtained (e.g., by ray tracing).

These mean that if the source beam is not focused at its waist, it appears as a modification of the $g$ and $\gamma$ parameters. So, with the interpretation of the parameters detailed previously and with the Rayleigh length $L_{0}$ of the focused beam, (1) can be used for more general cases than the examples presented in [9].

\section{B. Fourier-transform spectral interferometry}

The CEP-variation measurements were carried out by the use of spectral interferometry (SI) [15]. SI has been proposed earlier for single-shot CEP analysis in an f-to-2f geometry utilizing frequency up-conversion [19]. Similarly, Gouy phase-shift measurements were carried out applying a supercontinuum [6]. However, it has been shown that there is no need for nonlinear optical phenomena for the CEP drift measurement, and SI can be used as a simple, linear optical method to get information on shot-to-shot CEP changes [20]. It can be applied even on picosecond-long pulses for CEP drift detection [21]. Spatial CEP distribution of a beam coming from a laser amplifier has also been studied using SI [22].

It is important to emphasize already at this point that this measurement method is relative and does not provide an "absolute" value of the phase. In the measurements where the CEP-stability of laser oscillators is the question [20,21], a preceding pulse in the pulse train serves as a reference. During the measurements in this work, the reference was a pulsed beam from the reference arm of a Mach-Zehnder interferometer, which served as a stable reference along a long propagation path, due to its collimation.

Before going into the details of the experiment, some theoretical points have to be considered regarding the phase obtained by using FTSI. As is known, by spectrally resolving the interference of the two beams coming from the two arms of an interferometer, a frequency-dependent intensity function can be detected which is of the form [15]

$$
I(\omega)=I_{\mathrm{sam}}(\omega)+I_{\mathrm{ref}}(\omega)+2 \sqrt{I_{\mathrm{sam}}(\omega) I_{\mathrm{ref}}(\omega)} \cos [\Delta \varphi(\omega)]
$$

In the interferometric signal of (4) $I_{\mathrm{sam}}(\omega)$ and $I_{\text {ref }}(\omega)$ are the intensity spectrum of the sample and reference beams, respectively, and $\Delta \varphi(\omega)$ is the phase difference between the two beams.

The $\Delta \varphi(\omega)$ relative spectral phase can be obtained from the spectrally resolved interference patterns by the Fouriertransform method [15]. The evaluation of the interferograms 
with the Fourier-transform method consists of three main steps, summarized also in Fig. 1. First, the inverse Fourier transform of the measured interferogram (see Fig. 1(a)) is taken, which yields a temporal signal (see Fig. 1(b)). In the temporal data $I(t)$ two peaks appear - due to the properties of the Fourier transform - corresponding to the pulse coming from the sample arm. Second, by Fourier transforming the relevant part of the such-retrieved temporal shape (see the region surrounded by dashed rectangle in Fig. 1(b)) one gets the complex spectrum of the sample arm. Finally, by taking the complex argument of the data one can get the $\Delta \varphi(\omega)$ phase difference (see Fig. 1(c)).

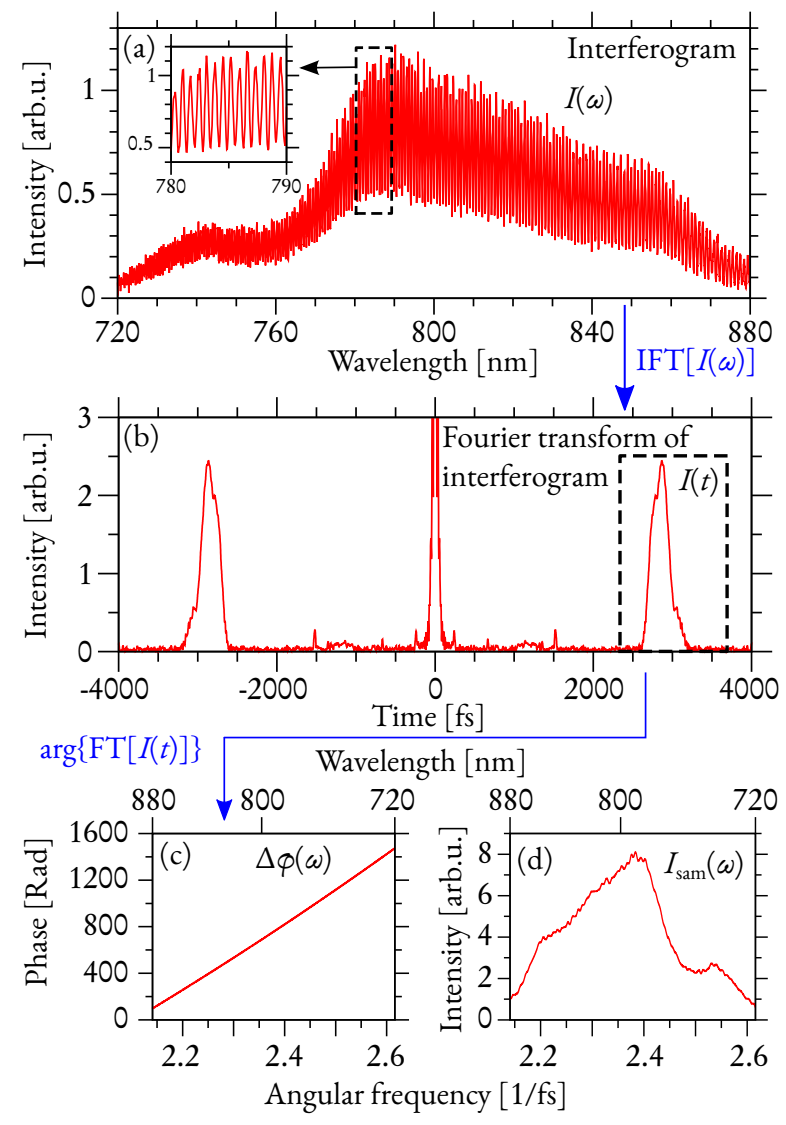

Fig. 1. The main steps of obtaining the complex spectrum with spectral interferometry applying the Fourier-transform method [15]. (a) A typical measured interferogram during this study. (b) The inverse Fourier transform of the interferogram. (c) The phase difference obtained by the selecting the relevant region from the temporal shape in (b) (highlighted by dashed rectangle), filling the other data with zero, and Fourier transforming the obtained data series. The phase is the complex argument of this data. (d) The measured sample arm spectrum by blocking the reference signal, and thus detecting only the first term in the right-hand side of (4).

By blocking the reference signal, the intensity spectrum of the sample beam can also be measured by the spectrometer. Measuring directly the sample spectrum (see Fig. 1(d)) is a more precise source for $I_{\text {sam }}(\omega)$ than the one that can be extracted from the interferogram with the Fourier-transform method.

\section{EXPERIMENTAL}

\section{A. Experimental setup}

The setup used for the experimental CEP analysis with SI can be seen in Fig. 2.

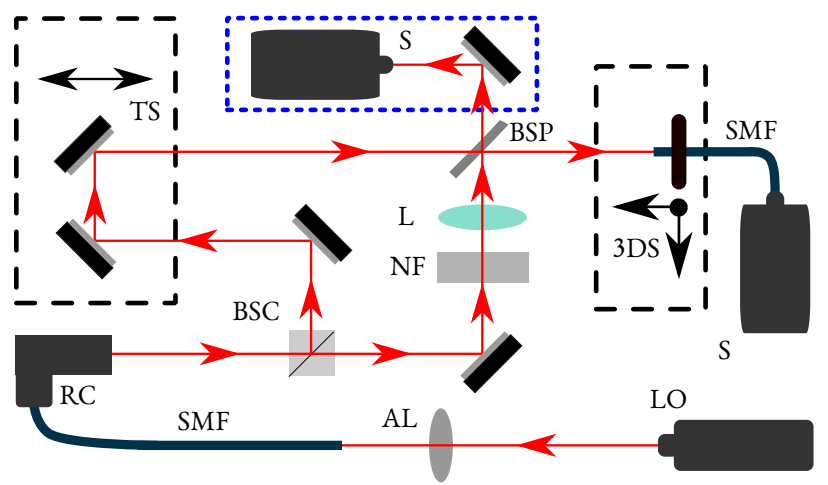

Fig. 2. The experimental setup. The basis is a Mach-Zehnder interferometer. The components are abbreviated according to the following: LO laser oscillator, AL achromatic lens, SMF single-mode fiber, RC reflective collimator, BSC beam-splitter cube, TS translator stage, NF neutral filter, L Lens, BSP beamsplitter plate, 3DS three-dimensional stage, $\mathrm{S}$ spectrometer.

The broadband light source was a Titanium:Sapphire laser oscillator (LO, Femtolasers Rainbow, $\lambda_{0}=800 \mathrm{~nm}$, bandwidth $\Delta \lambda=300 \mathrm{~nm})$. The beam of the laser was coupled into a singlemode fiber (SMF, Thorlabs-Nufern 780-HP, $5 \mathrm{~m}$ long) by an achromatic lens (AL, Thorlabs AC254-030-B-ML), and coupled out by a reflective collimator ( $\mathrm{RC}$, Thorlabs RC08) at the other end. This step was necessary to obtain a high-quality beam so the measurements can be compared with theoretical expression obtained for pulsed Gaussian beams. The collimated beam was sent through a broadband beam-splitter cube (BSC) to get identical copies of the pulsed beam in the sample and reference arms of the Mach-Zehnder type interferometer. While the length of the reference arm was adjustable by the translator stage (TS), the sample arm was fixed in length and contained a series of neutral filters (NF) and an achromatic lens (L, Thorlabs AC254200-B-ML, $200 \mathrm{~mm}$ focal length). The neutral filters were needed because without them the intensity of the signal from the sample arm would have been several orders of magnitude larger than the signal from the reference arm due to focusing, making measurements impossible. The beams from the two arms were combined using a beam-splitter plate (BSP). The BSP was set in such a way that the focused beam coming from the sample arm was reflected from it and the reference beam passed through its material. This was necessary to avoid possible distortions by focusing through a dispersive component. Finally the interference of the combined pulsed beams was spectrally resolved by a spectrometer (S, Ocean Optics HR-4000) coupled with a single mode fiber (SMF, same type as the previous one, $1 \mathrm{~m}$ long). The input end of the SMF was placed on a three-dimensional delay stage (3DS, Newport) to allow spatial scanning. Using a fiber for detection instead of the entrance slit of the spectrometer gives a better spatial resolution [23], as the radius of the core is $\sim 2 \mu \mathrm{m}$ compared with the $10 \mu \mathrm{m} \times 200 \mu \mathrm{m}$ size of the slit. The measurement range of the spectrometer $(710-895 \mathrm{~nm})$ was smaller then the total bandwidth of the beam, but this does not affect the measurement results, as explained later. Separated 
in a blue dotted region in Fig. 2 an additional, fixed-position spectrometer is highlighted, which was used for monitoring the stability of the interferometer in preliminary measurements.

\section{B. Interferometric data acquisition and evaluation}

A question that can be raised is the phase stability during the measurements, primarily determining the precision of the results. The phase changes of the reference beam due to propagation are negligible, as the reference beam is well collimated and the measurements were carried out in regions only a few millimeters long. Errors originating from the resolution of the spectrometer were minimized by applying short delays between the two arms, which made high precision recording of the spectral modulation available (the data series in Fig. 1(a) consists of 3648 points). Long term drifts, like the effect of temperature changes and air movement, or instability of the interferometer, were not affecting the measurements. This was confirmed by preliminary investigations applying simultaneous recording of the interference during measurement by a fixed-position spectrometer placed at the other output of the interferometer (see the blue dotted region in Fig. 2), giving information on the temporal variations of the phase. The laser source was not phase stabilized and the recording was multiple shot, but these do not affect the results as the method measures relative phases and the interferometer was proved to be stable.

It is visible from the temporal shape in Fig. 1(b) that due to the neutral filters and the lens material in the sample arm compared to the empty reference arm (see Fig. 2) - the directly evaluated pulse shape using the measured $I_{\text {sam }}(\omega)$ and $\Delta \varphi(\omega)$ is several-hundred femtoseconds long. However, in applications there are no filters before the focusing element in the experimental setup. For this reason a fixed spectral phase was subtracted from the measured $\Delta \varphi(\omega)$. This also coincides with the considerations of the simulations, where the material dispersion of a slab with the same properties as the lens central part is assumed to be pre-compensated. So, during the evaluation, the measured $\Delta \varphi(\omega)$ spectral phase in the focal point was fitted with a thirdorder polynomial, and this polynomial was subtracted from the phase at all measurement points, exactly the same way it was done in previous and current simulations [9]. Choosing the compensating phase as a polynomial is advantageous because by subtracting such arbitrary spectral phase from the measured $\Delta \varphi(\omega)$ during evaluation provides fully equivalent data with non-relative phase measurements carried out by applying a phase-shaper, e.g. an acousto-optic programmable dispersive filter [24], on the input beam. This is also the reason why it is not relevant that the reference pulse is not characterized. With a similar argumentation the pulsed beam reaching the focusing element can be thought of as transform-limited pulse. The key is that subtracting such a compensating phase is physically the same as applying a homogenous phase modification along the cross section of the beam in a point where there are no strong spatio-temporal couplings present [25], that is before the focusing element. So, evaluating the measured data this way gives a physically meaningful result.

To determine the CEP in a spatial point with the obtained experimental data - the directly measured $I_{\text {sam }}(\omega)$ intensity spectrum (Fig. 1(d)) and the modified value of the phase $\Delta \varphi(\omega)$ (Fig. 1(c)) - an inverse Fourier transform is applied, to access the temporal shape of the field. From the temporal shape the CEP is extracted by calculating the temporal phase at the instant of time when the pulse envelope reaches its maximum, to obtain the CEP with the same definition as in [9]. This method was repeated on measurement data recorded in several points along lines parallel to the optical axis.

\section{RESULTS}

\section{A. Beam characterization}

Before carrying out the CEP-change measurements in the focal region, the source beam had to be characterized to make comparisons with theoretical predictions possible. As already mentioned in the theoretical summary, two wavelength-dependent parameters are relevant regarding the CEP changes: the size of the beam and the radius of curvature of the wave front reaching the focusing element $[9,17]$. The latter is used to obtain data on the wavefront behind the focal element using ray tracing.

The reflective collimator in the measurement setup (see Fig. 2) would produce a broadband beam with wavelengthindependent beam size in the case that the fiber-supported modes were wavelength independent. However, the mode field radius (MFR) of guided modes of fibers are known to depend on frequency [26]. So, to get a proper characterization of the collimated beam, the following method was applied which combined experimental data with the results of beam-propagation simulations. First, spectral measurements were carried out in lines perpendicular to the axis of propagation. By moving a spectrometer step by step crossing a transverse section of the laser spot, a segment of the intensity distribution of the beam can be obtained for different wavelengths. The results of such a measurement can be seen in Fig. 3 depicting typical data series for three wavelengths. Fitting Gaussian curves to these points (black lines for each wavelength in Fig. 3), the wavelength dependence of beam radius (intensity $1 / \mathrm{e}^{2}$ ) can be obtained at a given distance from the collimator.

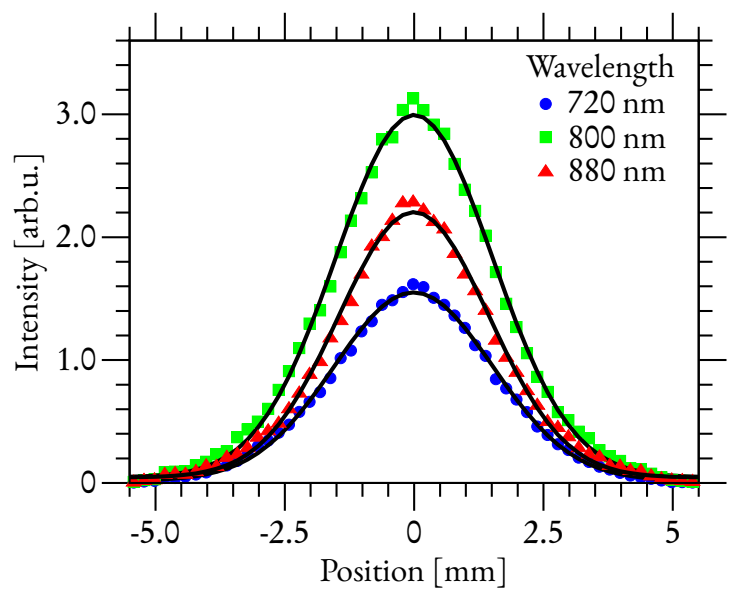

Fig. 3. Typical measurement results obtained by scanning a segment of a broadband beam with a spectrometer. The black curves are the fitted Gaussian curves. The intensity of the beam profiles has been scaled for the different wavelengths for better visibility.

To retrieve wave front curvature data the beam-size measurements were used to deduce information on the wavelength dependence of the MFR in the fiber, then the wave front was simulated by propagating the beam from the fiber through the reflective collimator to the distance in question using commercial beam propagating software. The wavelength-resolved beam segments were recorded several times at different distances from 
the collimator output, then these beam-radius data were compared with the ones obtained by beam propagation software assuming various mode-size values at the output of the fiber. The result of this analysis, so the wavelength dependence of MFR at the fiber end can be seen in Fig. 4 with black continuous curve. The error estimates are deduced from repeating the beam-size measurement at several distances from the collimator output and applying the above MFR-determination method. The resulted MFR curve can be fitted well with the Petermann II formula (see Fig. 4 red dashed curve) [26], which describes the MFR of single mode step-index fibers in this wavelength regime with $1 \%$ accuracy [27]. The fit implies a core radius of $2.07 \mu \mathrm{m}$ and numerical aperture of 0.11 , which agrees well with values given by the manufacturer, $2.2 \mu \mathrm{m}$ and 0.13 , respectively.

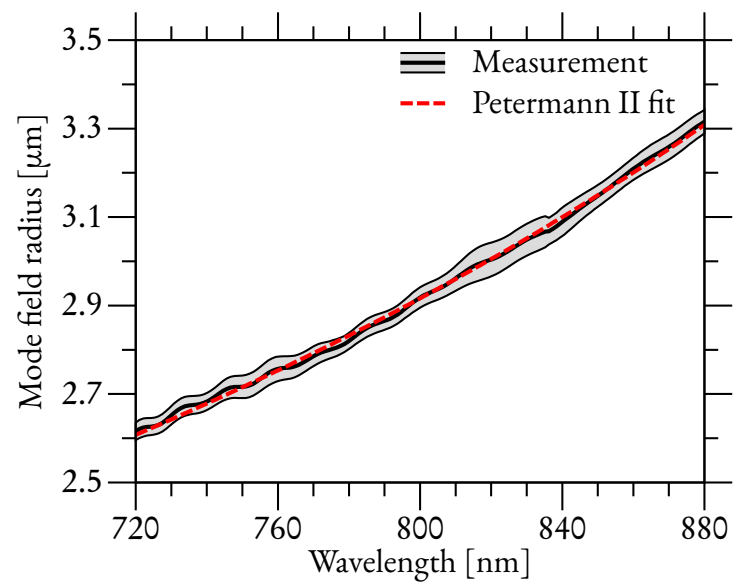

Fig. 4. The measured wavelength dependence of the mode field radius of the single mode fiber (black continuous curve) and the fitted approximate theoretical dependence (red dashed curve) based on the Petermann II formula [26].

The relevant properties in this study are the beam size and wave-front curvature values at the focusing element. The wavelength dependence of the beam size $70 \mathrm{~cm}$ away from the output of the reflective collimator can be seen in Fig. 5 with black curve. This is the position where the lens was placed in the interferometric setup during the CEP measurements (see Fig. 2).

The measured beam size variation with wavelength is primarily a consequence of the frequency dependence of the MFR of the fiber. The error estimates in this case were based on repeating the measurement with two different spectrometers (Ocean Optics HR-4000, Avantes Avaspec-3648). As a comparison, the simulated beam size is shown in Fig. 5 with red dashed curve. The calculated data is a result of the beam propagation calculations assuming the MFR variation depicted in Fig. 4.

The wave front curvature is also important to be able to simulate the electric field and the CEP variations in the focal region. However, the direct measurement in this case was not possible. Instead, based on the MFR results, the radii of the monochromatic wave fronts at the focusing element were calculated using beam propagation software. The results of the wave front calculations can be seen in Fig. 6 .

The wave front curvatures behind the lens are needed for the analytical formula (1) and also for numerical calculations in off-axis points. Propagation through lens material can perfectly be modeled with ray optics, so the paraxial wave front behind the lens was calculated with ray tracing based on the measured

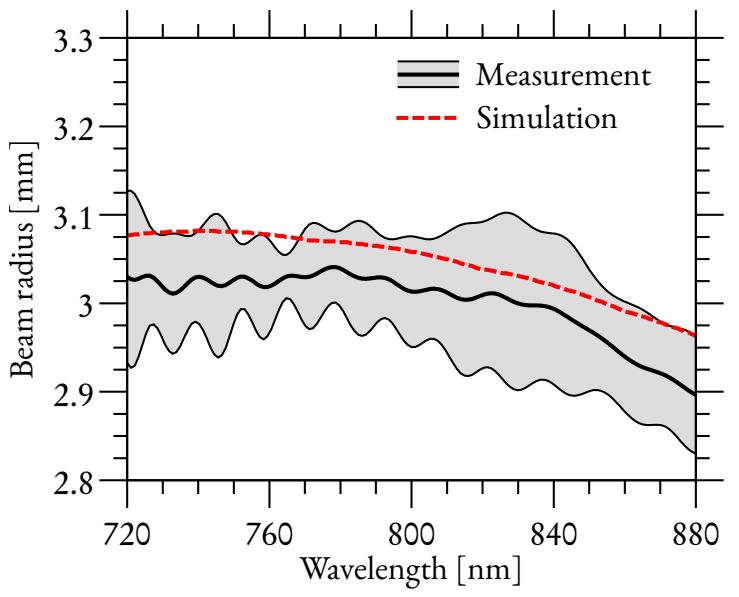

Fig. 5. The wavelength dependence of the beam radius (intensity $\left.1 / \mathrm{e}^{2}\right)$ at $70 \mathrm{~cm}$ distance from the output of the reflective collimator (see Fig. 2). The simulated curve is the one obtained with commercial beam propagation software assuming a mode field radius of the single mode fiber plotted in Fig. 4.

phase front data before the lens depicted in Fig. 6.

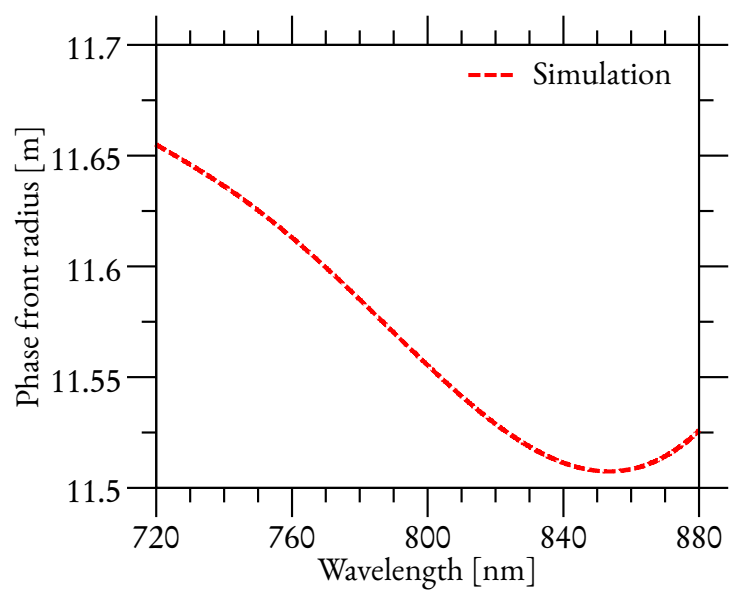

Fig. 6. The simulated wavelength dependence of wave front radius at the position of the focusing lens obtained using commercial beam propagation software and the estimated mode field radius in Fig. 4.

\section{B. Variations of the carrier-envelope phase in the focal region}

Fig. 7 depicts results of measurements carried out with FTSI using the evaluation method detailed previously in the Theoretical background and Experimental sections.

It can be seen in the three cases of Fig. 7 that the CEP changes differently during propagation along lines parallel to the optical axis, depending how far this line is from the center of symmetry. The change of the CEP is considered as relative values with respect to the CEP value in the focus. As phase values are not absolute values the reference point is arbitrary. The black continuous curve in Fig. 7 shows a phase change corresponding to the Gouy phase shift, i.e., a function expressed by the first term in the right-hand side of (1). The deviations of the measured CEP variations from Gouy phase are thus clearly visible, being 


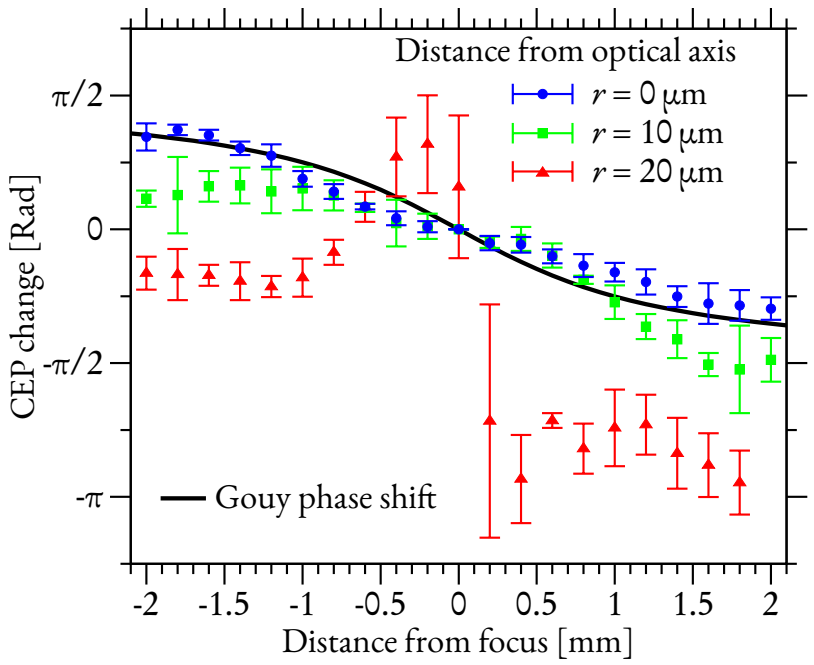

Fig. 7. The measured CEP change of an ultrashort pulse during propagation through focus along lines parallel to the optical axis. The black continuous curve shows the Gouy phase shift highlighting the differences of the measured phase changes from it.

more pronounced as the line of propagation is farther away from the optical axis.

The CEP-variation curves presented in Fig. 8 are simulation results corresponding to the measurement plotted in Fig. 7 . The numerical simulation curves plotted in Fig. 8 are based on the method presented in [9], but extended to off-axis points. The numerical approach was necessary because (1) is only valid for on-axis propagation. Away from the optical axis one has to consider transverse phase variations [28]. It is possible to obtain analytical expression for CEP changes off-axis, but they are interpreted on caustic surfaces and not along lines parallel to the axis of propagation [8].

Going a little bit more into details regarding the numerical calculations, the beam properties obtained from the measurements (presented above and depicted in Figs. 5 and 6) served as input parameters for the simulations. Using them the wave fronts of the monochromatic components behind the focusing element were calculated using an own-written ray-tracing software. Then the electric field in the vicinity of the focus was obtained using scalar diffraction theory, by evaluating the Kirchhoff diffraction integral [18]. The simulation takes into account the dispersion of the lens material, the truncation of the lens aperture, the variable thickness of the lens and the spherical aberration related to it. The simulation allows the same "virtual" dispersion compensation as the one applied during evaluation of measurement data. As it can be seen in Fig. 8, the simulation gives $C E P$ variations that follow a similar trend to the CEP measurements for all radii. This qualitative agreement supports the determining effect of the two wavelength-dependent parameters suggested by the analytical model. The shaded regions around the CEP curves in Fig. 8 are aimed to provide an estimation on the uncertainty in the comparison due to the fact that measurements were carried out in a circular area with finite size given by the size of the fiber core, while the simulations present values in an infinitely small point. This error estimation also takes into account that the positioning of the fiber end has a precision of $\sim 1 \mu \mathrm{m}$. The region given in the plots of Figs. 7

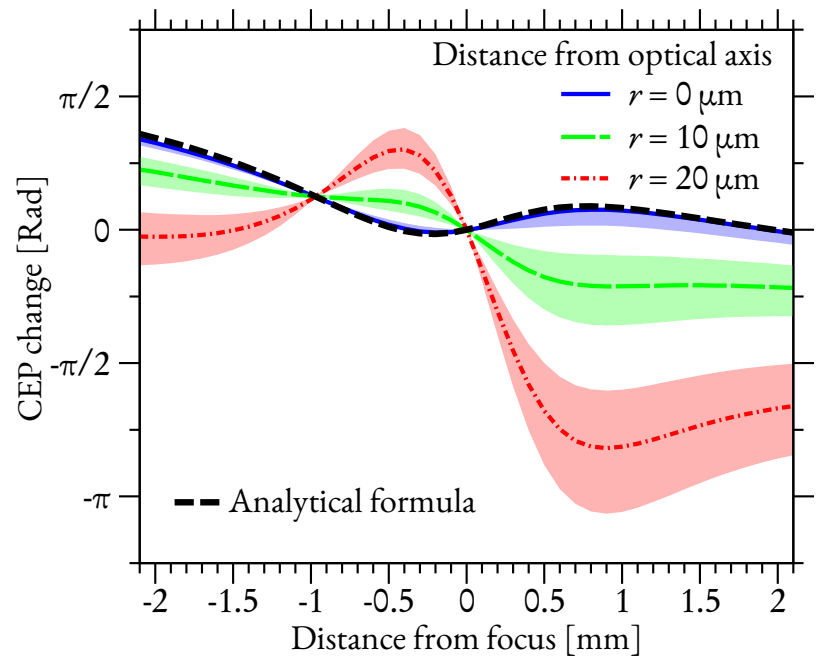

Fig. 8. The simulated CEP change corresponding to the same $r$ values as in Fig. 7. The analytical curve is the one calculated using (1), with necessary parameters $g$ and $\gamma$ deduced from the measurements presented in Figs. 5 and 6 . The shaded region around the lines shows numerical uncertainty estimates because of the fact that the core of a fiber has a finite size, and the curves correspond to simulation results in an infinitely small point.

and 8 are slightly bigger than the area that is most important in experiments as the Rayleigh length and the beam waist radius at the carrier wavelength $(800 \mathrm{~nm})$ are $\sim 1 \mathrm{~mm}$ and $\sim 16 \mu \mathrm{m}$, respectively. These values are confirmed by both simulation and measurement.

By comparing the results of Figs. 7 and 8 it is visible that there are some differences between measurement and simulation data. These discrepancies are a result of uncertainties in the parameters of the simulation. An evident inaccuracy carried by the simulation input is the wave-front curvature data, as these data are not directly measured, instead they are simulated based on measurement of other beam properties. To check the effect of the spherical wave-front radius, the focusing simulations were carried out in the form of a fitting algorithm on the measured data with the variation of wave-front curvature with wavelength being the fitting parameter. This algorithm did not provide better correspondence between simulation and measurement. Using simulated data for the wave fronts also means that an ideal, spherical wave front is assumed, compared to the real one, which is probably not ideally spherical and may be distorted. Analyzing the color-dependent distortions of the phase fronts and its effects on the phase properties are outside the scope of this work. However, these differences between simulation and measurement caused by the non-direct acquisition of beam properties could be decreased by applying a method that is capable of precise, frequency-resolved wave front measurement [29-31].

For comparison with the analytical, on-axis formula motivating this work the pulse shape changes during propagation is an important issue, as the constancy of the pulse envelope is a requirement for the validity of (1). The measurement and evaluation methods make it available to check the non-reshaping envelope prerequisite of formula (1). The envelope shapes obtained by applying inverse Fourier transform on the phase- 
compensated measurement data can be seen in Fig. 9 in several points close to the focus.

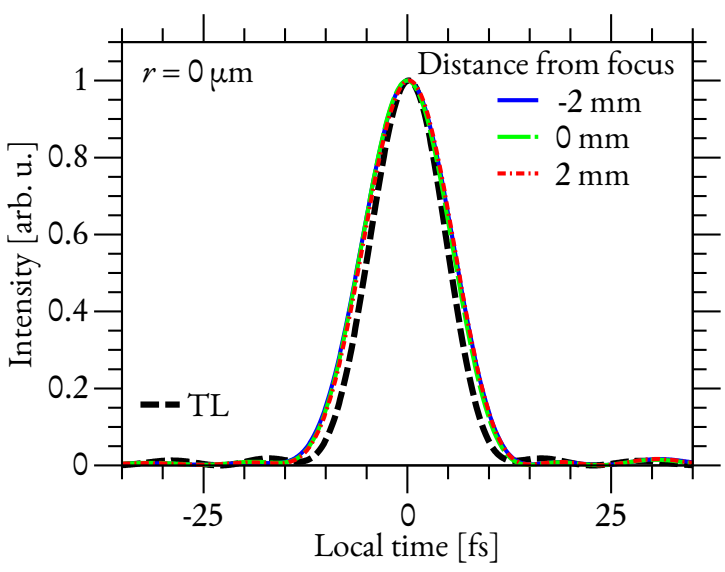

Fig. 9. Normalized temporal envelope shapes of the pulse in the focal region calculated using the measured, compensated complex spectral data. The overlapping colored curves show the pulse shapes in three points along the optical axis at different distances from the focal point. The black short-dashed curve represents the transform-limited (TL) pulse shape given by the amplitude spectrum measured in the reference arm.

It can be seen in Fig. 9 that during on-axis propagation (depicted at $-2,0$ and $2 \mathrm{~mm}$ from the focal point) the shape of the temporal envelope does not change. Of course, there are differences from the transform-limited (TL) shape of the pulse based on the measured reference-arm amplitude spectrum (black shortdashed curve) due to propagation effects. So the conditions of formula (1) are fulfilled in our experimental conditions. This also means that the CEP changes measured are independent of the exact shape of the amplitude spectrum, which was verified by applying artificial modification to it. This is also the reason why it is irrelevant in this case that the spectrometer does not cover the whole bandwidth of the broadband source. For a case when the envelope shape changes during propagation, using a spectrometer that covers the whole spectrum of the radiation is necessary. Calculating the $g$ and $\gamma$ parameters from the measured properties (Figs. 5 and 6 ) yield $g=1.413$ and $\gamma=0.673$, with which the analytical formula (1) fits the numerical results (see Fig. 8 thick black short-dashed curve).

\section{CONCLUSIONS}

As a conclusion, measurements based on spectral interferometry were presented in this work which studied the CEP changes occurring during propagation through the focal region. These show that the CEP-properties can change in the volume surrounding the focal spot, and these variations can relevantly differ from Gouy phase shift. The CEP changes were also analyzed theoretically, and the simulation results show similar variations of the CEP as the ones obtained by the SI measurements. The wavelength-dependent properties of the pulsed beam were also characterized, which are the main reason of the measured CEP changes, as demonstrated in this work. Based on the data presented it is important to consider the effects of wave propagation even in such CEP-dependent light-matter interaction phenomena, where the substance is so sparse that the influence of the material on the pulsed light beam can be neglected. The re- sults also suggest that in CEP determination methods based on CEP-dependent phenomena happening in a larger volume the volumetric integration has to be considered.

\section{ACKNOWLEDGMENTS}

The authors are grateful for the help of the colleagues at the TeWaTi laboratory of the Department of Optics and Quantum Electronics, University of Szeged. The project was partially funded by TÁMOP-4.2.2.D-15/1/KONV-2015-0024 - 'ELITeamEstablishment of the ELI Institute at the University of Szeged: foundation of interdisciplinary research in the field of lasers and their applications', which is supported by the European Union and co-financed by the European Social Fund. B.M. is grateful for project TÁMOP 4.2.4.A/2-11-1-2012-0001 ‘National Excellence Program'. M.A.P. acknowledges support from Projects No. MTM2012-39101-C02-01 and No. FIS2013-41709-P of the Spanish Ministerio de Economía y Competitividad.

\section{REFERENCES}

1. F. Krausz and M. Ivanov, "Attosecond physics," Rev. Mod. Phys. 81, 163-234 (2009).

2. C. Manzoni, O. D. Mücke, G. Cirmi, S. Fang, J. Moses, S.-W. Huang, K.-H. Hong, G. Cerullo, and F. X. Kärtner, "Coherent pulse synthesis: towards sub-cycle optical waveforms," Laser \& Photon. Rev. 9, 129-171 (2015).

3. G. Cerullo, A. Baltuška, O. Mücke, and C. Vozzi, "Few-optical-cycle light pulses with passive carrier-envelope phase stabilization," Laser \& Photon. Rev. 5, 323-351 (2011).

4. F. Lindner, G. G. Paulus, H. Walther, A. Baltuska, E. Goulielmakis, M. Lezius, and F. Krausz, "Gouy phase shift for few-cycle laser pulses," Phys. Rev. Lett. 92, 113001 (2004).

5. T. Tritschler, K. D. Hof, M. W. Klein, and M. Wegener, "Variation of the carrier-envelope phase of few-cycle laser pulses owing to the gouy phase: a solid-state-based measurement," Opt. Lett. 30, 753-755 (2005).

6. Z. Wang, Z. Zeng, R. Li, and Z. Xu, "Measurement of gouy phase shift by use of supercontinuum spectral interference," Chin. Opt. Lett. 5, S183-S185 (2007).

7. Y.-H. Zhou, H.-B. Jiang, and Q.-H. Gong, "The carrier-envelope phase of focused few-cycle laser pulses," Chinese Phys. Lett. 23, 110 (2006).

8. M. A. Porras, "Characterization of the electric field of focused pulsed gaussian beams for phase-sensitive interactions with matter," Opt. Lett. 34, 1546-1548 (2009).

9. M. A. Porras, Z. L. Horvath, and B. Major, "On the use of lenses to focus few-cycle pulses with controlled carrier-envelope phase," Appl. Phys. B: Lasers Opt. 108, 521-531 (2012).

10. T. Popmintchev, M.-C. Chen, P. Arpin, M. M. Murnane, and H. C Kapteyn, "The attosecond nonlinear optics of bright coherent x-ray generation," Nature Photon. 4, 822-832 (2010).

11. T. Wittmann, B. Horvath, W. Helml, M. G. Schaetzel, X. Gu, A. L. Cavalieri, G. G. Paulus, and R. Kienberger, "Single-shot carrier-envelope phase measurement of few-cycle laser pulses," Nature Phys. 5, 357362 (2009).

12. T. Rathje, A. M. Sayler, S. Zeng, P. Wustelt, H. Figger, B. D. Esry, and G. G. Paulus, "Coherent control at its most fundamental: Carrierenvelope-phase-dependent electron localization in photodissociation of a $\mathrm{H}_{2}^{+}$molecular ion beam target," Phys. Rev. Lett. 111, 093002 (2013).

13. M. J. Abel, T. Pfeifer, A. Jullien, P. M. Nagel, M. J. Bell, D. M. Neumark, and S. R. Leone, "Carrier-envelope phase-dependent quantum interferences in multiphoton ionization," J. Phys. B: At., Mol. Opt. Phys. 42, 075601 (2009).

14. P. Dietrich, F. Krausz, and P. B. Corkum, "Determining the absolute carrier phase of a few-cycle laser pulse," Opt. Lett. 25, 16-18 (2000).

15. L. Lepetit, G. Chériaux, and M. Joffre, "Linear techniques of phase measurement by femtosecond spectral interferometry for applications in spectroscopy," J. Opt. Soc. Am. B 12, 2467-2474 (1995). 
16. L. G. Gouy, "Sur une propriete nouvelle des ondes lumineuses," C. R. Acad. Sci. Paris 110, 1251 (1890).

17. B. Major, Z. L. Horváth, and M. A. Porras, "Phase and group velocity of focused, pulsed gaussian beams in the presence and absence of primary aberrations," J. Opt. 17, 065612 (2015).

18. M. Born and E. Wolf, Principles of Optics (Cambridge University Press, 1999), 7th ed.

19. M. Kakehata, H. Takada, Y. Kobayashi, K. Torizuka, Y. Fujihira, T. Homma, and H. Takahashi, "Single-shot measurement of carrierenvelope phase changes by spectral interferometry," Opt. Lett. 26 1436-1438 (2001).

20. P. Jójárt, A. Börzsönyi, B. Borchers, G. Steinmeyer, and K. Osvay, "Agile linear interferometric method for carrier-envelope phase drift measurement," Opt. Lett. 37, 836-838 (2012).

21. P. Jójárt, A. Börzsönyi, V. Soskov, F. Zomer, R. Chiche, E. Cormier, and K. Osvay, "Carrier-envelope phase drift measurement of picosecond pulses by an all-linear-optical means," Opt. Lett. 39, 5913-5916 (2014)

22. Z. Yu and Y. Weng, "Spatial distribution of carrier-envelope phase for femtosecond pulsed laser beam profile determined by asymmetric spectral interferometry," Opt. Lett. 35, 2275-2277 (2010).

23. P. Bowlan, P. Gabolde, M. A. Coughlan, R. Trebino, and R. J. Levis, "Measuring the spatiotemporal electric field of ultrashort pulses with high spatial and spectral resolution," J. Opt. Soc. Am. B 25, A81-A92 (2008).

24. P. Tournois, "Acousto-optic programmable dispersive filter for adaptive compensation of group delay time dispersion in laser systems," Opt. Commun. 140, 245-249 (1997).

25. S. Akturk, X. Gu, P. Bowlan, and R. Trebino, "Spatio-temporal couplings in ultrashort laser pulses," J. Opt. 12, 093001 (2010).

26. K. Petermann, "Constraints for fundamental-mode spot size for broadband dispersion-compensated single-mode fibres," Electron. Lett. 19, 712-714 (1983).

27. R. Paschotta, Encyclopedia of laser physics and technology (WileyVCH Berlin, 2008). (https://www.rp-photonics.com/encyclopedia.html).

28. B. S. Davis and L. Kaplan, "Transverse phase variation of a gaussian beam," J. Opt. 15, 075706 (2013).

29. E. Frumker, G. G. Paulus, H. Niikura, D. M. Villeneuve, and P. B. Corkum, "Frequency-resolved high-harmonic wavefront characterization," Opt. Lett. 34, 3026-3028 (2009).

30. D. R. Austin, T. Witting, C. A. Arrell, F. Frank, A. S. Wyatt, J. P. Marangos, J. W. Tisch, and I. A. Walmsley, "Lateral shearing interferometry of highharmonic wavefronts," Opt. Lett. 36, 1746-1748 (2011).

31. Z. Guang, M. Rhodes, M. Davis, and R. Trebino, "Complete characterization of a spatiotemporally complex pulse by an improved single-frame pulse-measurement technique," J. Opt. Soc. Am. B 31, 2736-2743 (2014) 